\title{
Champ géomagnétique et dynamique du noyau terrestre
}

Nicolas Gillet (nicolas.gillet@univ-grenoble-alpes.fr)

Institut des Sciences de la Terre (Univ. Grenoble Alpes, Univ. Savoie Mont Blanc, IRD, UGE) 1381 rue de la Piscine, BP 40700, 38058 Grenoble Cedex 9

\section{Le champ géomagnétique}

est principalement généré par

les mouvements de métal liquide

dans le noyau de la Terre

par « effet dynamo », 3000 km

sous nos pieds. Son évolution

est suivie en continu

au sol et, depuis vingt ans,

par des satellites qui nous apportent

des informations sans précédent.

Notre compréhension

de la " géodynamo » a évolué

ces dernières années,

grâce aussi aux importantes

avancées des calculs numériques.

L'information portée par

les mesures magnétiques,

couplée à celle des simulations

hautes performances, nous aide

à reconstituer la dynamique

dans le noyau. Les champs

d'applications sont vastes,

la dynamique du noyau affectant

par exemple la durée du jour

ou le champ magnétique

gouvernant la météo de l'espace.
Le champ magnétique terrestre conditionne l'interaction de notre planète avec le flux de particules en provenance du Soleil (ou vent solaire). Les fluctuations géomagnétiques qui en résultent induisant des courants électriques dans le sol, il interfere alors avec notre civilisation. Ainsi, des tempêtes magnétiques ont été à l'origine de perturbations dans les télécommunications, et de pannes électriques étendues sur des milliers de $\mathrm{km}$ (comme celle, spectaculaire, de 1989 au Québec).

Le champ géomagnétique est aussi un facteur clé des aurores polaires. Partout autour de nous, il est utilisé par une partie de la faune pour s'orienter. C'est finalement un outil géophysique puissant : la mesure du champ magnétique enregistré dans la croute terrestre au fil des âges géologiques étaye par exemple la théorie de la tectonique des plaques.

\section{Observations géomagnétiques}

Sur Terre, un magnétomètre mesure une somme de contributions, soit internes (provenant du noyau liquide ou de la croute terrestre), soit externes (provenant de la magnétosphère à une distance de quelques rayons terrestres, ou de l'ionosphère à quelques centaines de $\mathrm{km}$ d'altitude). Le champ dynamo en provenance du noyau liquide, d'amplitude $\approx 50 \mu \mathrm{T}$ à la surface terrestre, représente la majeure partie de l'énergie magnétique mesurée. Cependant, près d'une zone volcanique, l'aimantation rémanente dans les roches peut être localement assez forte pour perturber une boussole et nous faire perdre le Nord. Dans un autre registre, les variations du champ magnétosphérique atteignent plusieurs centaines de nanoteslas (nT) lors de tempêtes magnétiques (résultat d'interactions entre le champ du noyau et le vent solaire).

Le géomagnétisme est une science relativement jeune : ainsi, il faut attendre le milieu du XIX ${ }^{\mathrm{e}}$ siècle et K.F. Gauss pour avoir la preuve que le champ géomagnétique est essentiellement d'origine interne à notre planète. Nous savons aujourd'hui qu'il provient d'écoulements de métal liquide dans le noyau externe, une coquille sphérique de $3485 \mathrm{~km}$ de rayon extérieur et de $1220 \mathrm{~km}$ de rayon intérieur, située au centre de notre planète (fig. 1). Le mécanisme de fonctionnement de cette "géodynamo " fut suggéré par J. Larmor en 1919 : de l'énergie cinétique est puisée dans les mouvements fluides pour générer des courants électriques (et donc un champ magnétique).

Quand on dit « liquide », il faut entendre fluide très peu visqueux, comme de l'eau, au contraire du manteau, roche de phase solide qui flue sur des temps géologiques. La connaissance des propriétés physiques, comme la densité du noyau, nous vient en premier lieu de l'étude des ondes sismiques qui traversent la Terre. On sait ainsi que le noyau est majoritairement composé de fer, plus un ou plusieurs éléments légers comme l'oxygène, le soufre, le silicium, le carbone et l'hydrogène. Cette connaissance est complétée par des calculs de chimie quantique et des expériences de laboratoire utilisant, par exemple, des presses à enclume 


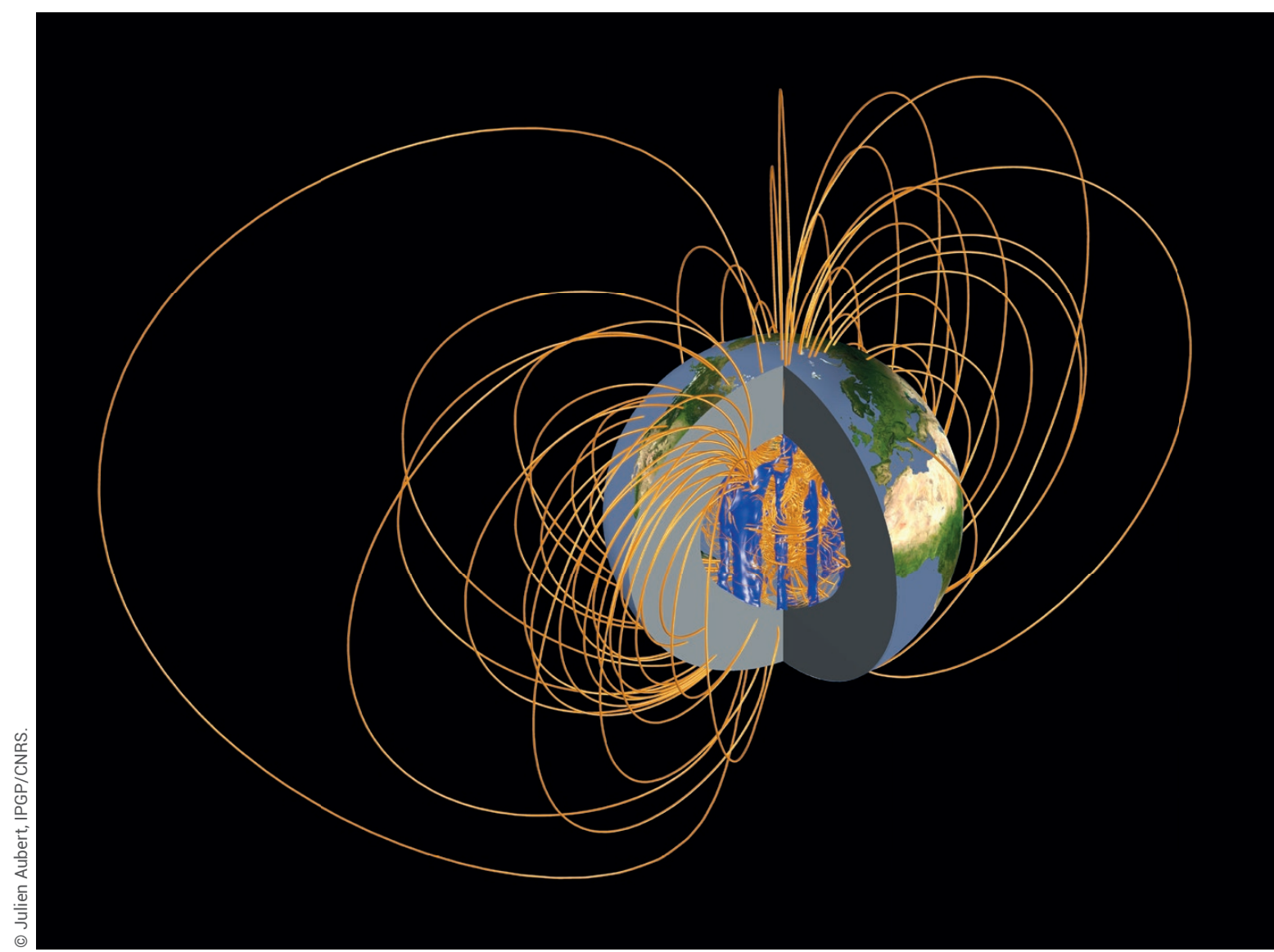

1. Une coupe de l'intérieur de la Terre, montrant le noyau externe sphérique. En orange, les lignes de champ magnétique et, en bleu, les colonnes fluides à l'origine du champ, alignées avec l'axe de rotation, du fait de la force de Coriolis.

de diamant ou des chocs laser. On peut ainsi contraindre les propriétés de la matière (compatibilité des divers éléments légers avec le fer liquide, conductivités électrique et thermique des alliages envisagés) sous ces conditions extrêmes de pression et de température $^{(a)}$. Connaitre ces ingrédients est crucial pour établir l'état convectif du noyau en déterminant, par exemple, le caractère stable ou non du gradient de densité sous sa surface externe.

La connaissance des écoulements dans le noyau se base sur les mesures de champ magnétique : depuis deux décennies, celles enregistrées depuis l'espace apportent des informations précieuses. Ainsi, depuis fin 2013, les trois satellites de la mission Swarm $^{(b)}$ de l'ESA permettent, entre autres, de séparer au mieux les sources magnétiques internes et externes à la Terre. Ces données complètent les mesures au sol effectuées depuis le milieu du XIX ${ }^{\mathrm{e}}$ siècle (et actuellement via le réseau Intermagnet) et les mesures historiques réalisées pour la navigation (cataloguées depuis la fin du
$\mathrm{XVI}^{\mathrm{e}}$ siècle). Sur des périodes millénaires et au-delà, le paléomagnétisme utilise des enregistrements indirects : extraits de poteries, fours, coulées de lave, ou encore sédiments contenant des matériaux susceptibles de "figer" le champ magnétique présent à l'époque de leur formation. On a pu ainsi découvrir les inversions des pôles magnétiques, qui s'opèrent sur une durée de quelques milliers d'années. La dernière a eu lieu il y a environ 800000 ans. Les traces les plus anciennes du champ magnétique terrestre (enregistrées dans des cristaux inclus dans des roches) remontent à au moins deux milliards d'années.

\section{La géodynamo}

La figure 1 montre les écoulements dans le noyau externe, permettant à la géodynamo de fonctionner. Ces écoulements génèrent des courants électriques, à l'origine du champ magnétique terrestre, à première vue dipolaire.

\section{Théorie}

Pour comprendre cet effet dynamo, il est particulièrement instructif de regarder la dérivée temporelle $\mathbf{B}^{\prime}(t)$ du champ magnétique $\mathbf{B}$ mesuré (fig. 2) : elle nous informe sur les écoulements de métal liquide sous la surface du noyau. En effet, en combinant les équations de l'électromagnétisme et la loi d'Ohm, on montre que la variation temporelle $\mathbf{B}(t)$ est régie par l'équation d'induction :

$$
\mathbf{B}^{\prime}(t)=\nabla \times(\mathbf{U} \times \mathbf{B}(t))+\eta \nabla^{2} \mathbf{B}(t)
$$

où $\eta \sim 1 \mathrm{~m}^{2} / \mathrm{s}$ est la diffusivité magnétique ${ }^{(\mathrm{c})}$ du noyau liquide et $U$ la vitesse du fluide dans le référentiel terrestre en rotation. Le premier terme à droite est une source (ou force électromotrice, f.e.m.) induite par le cisaillement des lignes de champ par l'écoulement fluide $\mathbf{U}$, et le second un puits (dissipation par effet Joule). Pour avoir une création pérenne d'un champ magnétique à partir de l'énergie cinétique, il faut que le terme source l'emporte. Pour cela, le nombre de Reynolds magnétique sans dimension $R_{m}=U D / \eta$ doit être très 


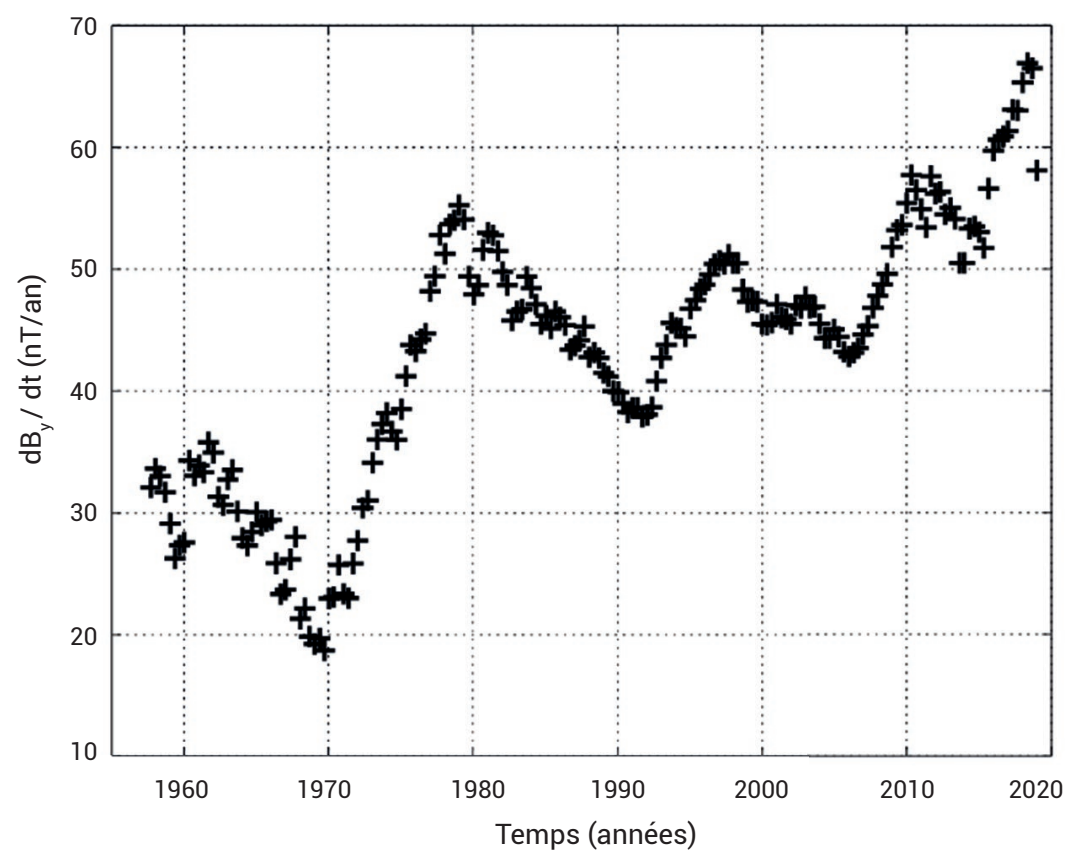

2. Série temporelle de la dérivée par rapport au temps de la composante $B_{y}$ (vers l'Est) du champ magnétique, mesurée à l'observatoire de Chambon-la-Forêt (France). Les changements abrupts de pente sont nommés "secousses magnétiques".

$\gg>$

supérieur à 1 : il caractérise en effet le rapport entre le temps caractéristique $T_{\eta}=D^{2} / \eta$ de dissipation d'une perturbation magnétique de taille $D$, et le temps $T_{U}=D / U$ pour que cette perturbation parcoure une distance de l'ordre de $D$ en étant entrainée à la vitesse $U$ du fluide.
Dans le cas terrestre, l'analyse dimensionnelle de l'équation (1) donne $U \sim D B$ ' $B$ noyau), $B \sim 30 \mu \mathrm{T}$ et $B^{\prime} \sim 100 \mathrm{nT} / \mathrm{an}$. Cela correspond à $R_{\mathrm{m}} \sim 1000>>1$, soit une dissipation magnétique bien inférieure à la f.e.m. aux grandes échelles spatiales. $\sim 10 \mathrm{~km} / \mathrm{an}$, pour $D \sim 3000 \mathrm{~km}$ (taille du
Pour aller plus avant vers une interprétation dynamique, il faut intégrer les effets des forces de Lorentz et de Coriolis, responsables des ondes d'Alfven et des ondes inertielles. Pour les premières, la force de rappel résulte du cisaillement des lignes de champ magnétique par l'écoulement. Non dispersives, elles se propagent le long des lignes de champ à une vitesse $U_{\mathrm{A}}=B /(\rho \mu)^{1 / 2}$ proportionnelle à l'intensité $B$ du champ magnétique ambiant $\left(\rho \sim 10^{4} \mathrm{~kg} / \mathrm{m}^{3}\right.$ est la densité du noyau et $\mu=4 \pi \times 10^{-7} \mathrm{H} / \mathrm{m}$ sa perméabilité magnétique). Les ondes inertielles sont, elles, liées à la force de Coriolis qui s'exerce sur les écoulements dans un fluide en rotation (à vitesse angulaire $\Omega=7,3 \times 10^{-3} \mathrm{~s}^{-1}$ dans le noyau). Leur période est de l'ordre de $T_{\Omega}=1 /(2 \Omega)$ (la journée). En quelques $T_{\Omega}$, elles organisent l'écoulement en colonnes alignées avec l'axe de rotation (fig. 1). Fortement dispersives, les ondes inertielles sont omniprésentes dans la mécanique des fluides dite géophysique (océan, atmosphère, noyaux planétaires, étoiles...).

Les caractéristiques des écoulements observés sont déterminées par les valeurs relatives des temps $T_{A}=D / U_{A}$ et $T_{\Omega}$ de propagation de ces ondes, et du temps de dissipation $T_{\eta}=D^{2} / \eta$, c'est-à-dire par les rapports :
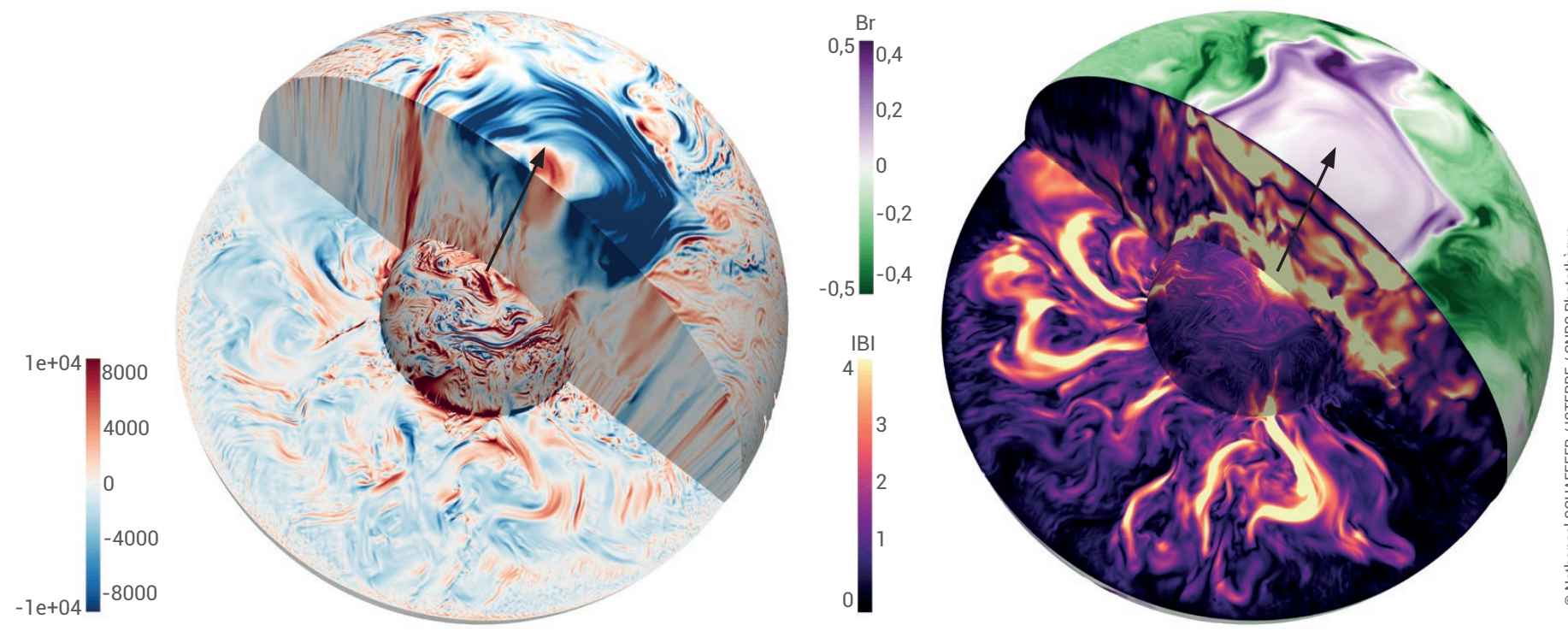

3. Coupe du noyau liquide dans une simulation numérique de la géodynamo [2].

À gauche : vitesse azimutale (en bleu vers l'Ouest, en rouge vers l'Est). La grande surface bleue représente une tornade géante de métal liquide de $1200 \mathrm{~km}$ de rayon, située sous le pôle Nord. Unité de mesure de la vitesse : mètres/an.

À droite : en volume, l'intensité |B| du champ magnétique (noir : nul, jaune : maximal), avec en surface le champ radial Br qui sort (violet) ou qui rentre (vert) du noyau. Unités : milliteslas.

La flèche noire indique l'axe de rotation de la planète. La sphère au centre représente la surface de la graine de fer cristallisé. 
- $L u=T_{\eta} / T_{A}=U_{A} D / \eta$

(nombre de Lundquist),

- Le $=T_{\Omega} / T_{A}=U_{A} /(2 \Omega D)$

(nombre de Lehner),

- $A l=T_{A} / T_{U}=U / U_{A}$

(nombre d'Alfven).

Dans le cas du noyau terrestre (D $3000 \mathrm{~km})$, on a $T_{\eta} \sim 300000$ ans $>>T_{\mathrm{U}} \sim 300$ ans $>>T_{\mathrm{A}} \sim 3$ ans $>>T_{\Omega} \sim 1$ jour, si bien que :

- Lu $\sim 10^{5}$ : une onde d'Alfven a largement le temps de se propager à travers le noyau avant d'être dissipée ;

- Le $10^{-4}$ : les ondes inertielles se propagent bien plus vite que les ondes d'Alfven. Les écoulements ont le temps de s'organiser en colonnes parallèles à l'axe de rotation ; - $A l \sim 10^{-2}$ : les ondes d'Alfven se propagent environ cent fois plus vite que le fluide.

\section{Études expérimentales}

Plusieurs projets expérimentaux ont cherché à reproduire l'effet dynamo, en forçant mécaniquement des écoulements de sodium liquide ${ }^{(\mathrm{d})}$. Atteindre des valeurs du nombre de Reynolds magnétique $R_{\mathrm{m}} \sim 50$ pour une taille caractéristique $D \sim 0,5 \mathrm{~m}$ (un volume $V \sim 0,1 \mathrm{~m}^{3}$ ), nécessite des vitesses $U \sim 10 \mathrm{~m} / \mathrm{s}$. Cela requiert une puissance $P_{U} \sim E_{U} / T_{U} \sim \rho V U^{3} /$ (2D), de l'ordre de $100 \mathrm{~kW}\left(E_{U} \sim \rho V U^{2} / 2\right.$ est l'énergie cinétique). L'effet dynamo a effectivement été observé expérimentalement pour des puissances de quelques centaines de $\mathrm{kW}$ [1] : d'abord à Riga et Karlsruhe en 2000 avec des écoulements très contraints, puis en 2006 à Cadarache (avec un effet ferromagnétique). Dans cette dernière expérience, il a par exemple été possible de reproduire les inversions de polarité du champ magnétique terrestre. D'autres projets, pourtant plus ambitieux (utilisation d'une sphère de 3 mètres de diamètre au Maryland), n'ont pas permis d'observer l'effet dynamo. Le diable se cache dans les détails : géométrie des écoulements, rôle des couches limites... Avec un champ généré de l'ordre de $10 \mathrm{mT}$, les dynamos expérimentales atteignent $A l \sim 30>>1$. Produire une dynamo ne suffit donc pas à modéliser le régime géophysique (où $A l<<1$ ), dans lequel la puissance à fournir est déterminée par l'énergie magnétique $E_{B} \sim V B^{2} /(2 \mu)$. Atteindre "seulement " $A l=0,1$ nécessite une puissance

$P_{B} \sim E_{B} / T_{U} \sim P_{U} / A l^{2} \approx 10 \mathrm{MW} .$.
On peut attaquer le problème géophysique sous un autre angle, en étudiant la turbulence et les ondes en présence d'un champ magnétique imposé. Toujours pour $D \sim 0,5 \mathrm{~m}$, atteindre $L u>>1$ (disons 100) nécessite un champ $B \sim \eta(\rho \mu)^{1 / 2} L u / D$ $\sim 0,7 \mathrm{~T}$, à maintenir sur le volume de l'expérience. Satisfaire dans le même temps à la condition $L e<<1$ (disons $10^{-2}$ ) requiert un taux de rotation $\Omega \sim \eta L u /\left(2 D^{2} L e\right) \sim$ $2000 \mathrm{rad} / \mathrm{s}$ ou 20000 tours/ minute. C'est pour aller en direction de ce régime qu'a été construite l'expérience DTS (Derviche Tourneur Sodium) à Grenoble, où des ondes d'Alfven, très affectées par la diffusion, ont été observées. Dans tous ces cas, le problème est que, dans un métal liquide, le champ magnétique diffuse bien plus vite que la quantité de mouvement, de sorte que soit la dissipation magnétique est trop forte ( $L u$ trop faible), soit le champ magnétique induit est trop faible ( $A l$ trop grand).

\section{Études numériques}

La voie numérique aussi est très exigeante : du fait de la rotation rapide de la planète, il est nécessaire d'intégrer les équations sur des durées très longues (quelques $10000 \mathrm{ans}$ ), tout en résolvant numériquement des fluctuations rapides (de période inférieure à la journée) et des couches limites très fines aux frontières du domaine (parois, interfaces). La puissance croissante des ordinateurs rend cependant possibles, dans certaines gammes de paramètres, des simulations reproduisant des caractéristiques du cas terrestre : dipôle prédominant, renversements des pôles magnétiques...

Pour donner une idée du défi auquel nous faisons face, simuler 5000 ans de l'évolution du noyau, même pour une viscosité dix millions de fois plus élevée qu'en réalité, nécessite aujourd'hui dix millions d'heures-cœur ${ }^{(e)}$, avec le code le plus performant [2]. Ces calculs montrent (fig. 3 gauche) un champ de vitesse du fluide très contraint par la rotation terrestre et organisé en colonnes alignées avec l'axe 


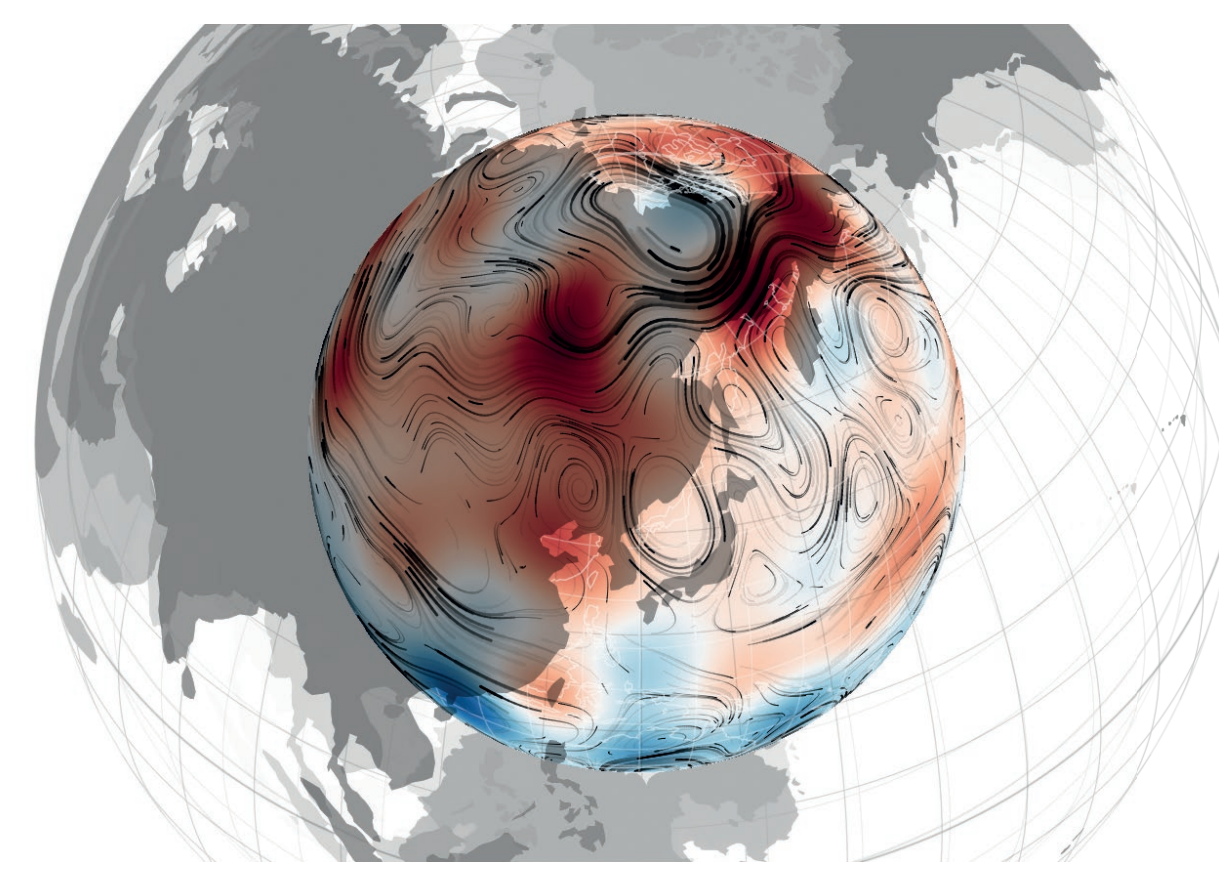

5. Assimilation de données géomagnétiques. Reconstitution, à partir de la mission Swarm, des écoulements (trajectoires noires, amplitude maximale de $40 \mathrm{~km} / \mathrm{an}$ ) et du champ magnétique (en rouge qui rentre dans le noyau, en bleu qui en sort, échelle de couleur $\pm 1 \mathrm{mT}$ ) à la surface du noyau externe en 2015. (https://geodyn.univ-grenoble-alpes.fr/).

$>>$

La première estimation du champ dans le noyau fluide provient de la détection d'ondes d'Alfven particulières : les ondes dites " de torsion ». Ces dernières s'organisent en tubes cylindriques alignés avec l'axe de rotation (fig. 4). À grande échelle, ce sont les seules ondes d'Alfven qui peuvent exister dans le noyau pour la raison suivante : dans la limite $L e<<1$, une perturbation dans le noyau serait normalement accommodée plus rapidement par les ondes inertielles que par les ondes d'Alfven. Cependant, la projection de la force de Coriolis sur les cylindres de torsion est nulle et ne créera donc pas d'ondes : ainsi, et seulement pour cette géométrie d'écoulements, c'est la force de Lorentz (à l'origine des ondes d'Alfven) qui interviendra. Décrites théoriquement par S. Braginski en 1970, les ondes de torsion permettent de sonder le champ magnétique dans lequel elles se propagent. Les ondes détectées à partir des mesures magnétiques traversent le noyau en quatre ans, soit une vitesse de phase $U_{\mathrm{A}} \sim 1000 \mathrm{~km} / \mathrm{an}$. Ceci implique un champ de quelques $\mathrm{mT}$ au sein du noyau liquide [3], environ dix fois plus fort qu'à sa surface.

ondes de torsion). Ce sont les ondes " magnéto-Coriolis ", pour lesquelles le terme de rappel fait intervenir à la fois la force de Coriolis et celle de Lorentz. Décrites dans les années 1960 par R. Hide et W. Malkus, elles se subdivisent pour $L e<<1$ en ondes rapides (ondes inertielles faiblement modifiées par B) et en ondes lentes. La période de ces dernières évolue comme $T_{A} / L e$, soit de l'ordre du millier d'années pour des perturbations de grande échelle. Dans ce schéma de pensée, il n'existe pas aux périodes interannuelles d'ondes non axisymmétriques de grande échelle.

C'est pourtant dans cette gamme de périodes qu'interviennent les «secousses magnétiques ". Ces variations abruptes de B' $(t)$, illustrées par la figure 2, sont de trop forte amplitude pour être expliquées uniquement par les ondes de torsion. Étant donné nos difficultés à observer et simuler les variations issues du noyau aux périodes plus courtes que quelques années, la question de savoir si les secousses sont des phénomènes isolés dans le temps, ou bien si elles existent en permanence, reste ouverte. Doit-on faire appel à la turbulence hydromagnétique en rotation pour les expliquer ? Des mécanismes ondulatoires de petite échelle en sont-ils la source?

La valeur de $U_{\mathrm{A}}$ est à comparer à la vitesse $U \sim 10 \mathrm{~km} /$ an caractéristique du fluide : leur rapport $A l \sim 10^{-2}$ indique que l'énergie magnétique est 10000 fois plus grande que l'énergie cinétique dans le noyau. La présence d'un champ magnétique d'une telle intensité est de prime-abord étonnante. En effet, l'ordre de grandeur $\mathrm{du}$ rapport entre force de Lorentz $\left(F_{\mathrm{L}} \sim B^{2} /(\mu D)\right)$ et force de Coriolis $\left(F_{\mathrm{C}} \sim 2 \rho \Omega U\right)$ indique $F_{\mathrm{L}} / F_{\mathrm{C}} \sim L e / A l \sim 10^{-2}$. La force magnétique n'apparait a priori pas si cruciale... Mais c'est sans compter avec le fait que la force de Coriolis ne travaille pas : c'est une force inertielle ou "fictive", perpendiculaire à la fois à l'axe de rotation et à la vitesse du fluide ; aussi, le système couplé des champs magnétique et de vitesse s'organise pour minimiser le travail de la force de Lorentz.

Cette énergie magnétique gigantesque est un ingrédient primordial qui distingue la dynamique du noyau de celle des enveloppes fluides superficielles comme l'océan et l'atmosphère, elles aussi dominées par la force de Coriolis. Pour de telles valeurs de $B$, apparait une famille d'ondes hydromagnétiques non axisymmétriques (i.e. avec des variations en azimuth, contrairement aux
Dans l'état actuel des ressources en calcul numérique, le régime où $A l<<1$ est très difficile à atteindre. Des tentatives émergent cependant, qui nécessitent de paramétrer les effets de la turbulence. Des secousses du type de celles montrées dans la figure 2 apparaissent alors et sont la signature de mécanismes ondulatoires. Comme c'est le cas avec les mesures magnétiques, elles sont plus fréquemment détectées dans la région équatoriale. Des panaches thermiques, en remontant depuis la surface de la graine solide, excitent des ondes d'Alfven structurées en colonnes alignées avec l'axe de rotation (du fait d'une force de Coriolis prédominante) et qui se propagent localement le long des lignes de champ magnétique [4]. Ces observations font écho à la découverte d'ondes magnéto-Coriolis de périodes interannuelles [5]. Dans les deux cas, ces ondulations semblent gouvernées par des structures de petite taille, tout en portant une signature à grande échelle qui pourrait correspondre aux secousses détectées dans les réseaux au sol et les mesures satellitaires. 


\section{Au-delà du noyau}

Les observations peuvent être combinées à l'intégration numérique d'un modèle dynamique dans le but de tirer au mieux profit de ces deux sources d'information. Si ces techniques, appelées " assimilations de données ", sont utilisées de manière opérationnelle en océanographie ou en météorologie, ce n'est que récemment qu'elles ont été introduites en géomagnétisme. La figure 5 montre une reconstitution par assimilation de données du champ de vitesse et du champ magnétique à la surface du noyau, à partir des mesures des satellites Swarm et de modèles simplifiés dérivés de simulations numériques de la géodynamo. Ces dernières ne sont pas encore utilisables de manière opérationnelle, mais pourraient permettre dans le futur d'interpréter les variations interannuelles observées $^{(\mathrm{f})}$

Les approches de ce type ont des applications possibles variées. Par exemple, le moment cinétique total de rotation de la Terre, somme de celui de ses différentes couches, doit rester constant. Si le moment cinétique d'une des couches fluides varie, cela doit donc être compensé par une variation en sens inverse du moment cinétique de la partie solide de la Terre. Ainsi, si le premier diminue, la vitesse de rotation de la Terre solide doit augmenter et la durée du jour sera réduite. Ces variations peuvent atteindre quelques millisecondes et être déterminées par interférométrie des ondes radio émises par les étoiles.

Aux périodes plus courtes que quatre ans environ, ce sont les variations du moment cinétique associé aux écoulements atmosphériques qui déterminent les variations de la durée du jour.

En revanche, les variations de plus longue période dépendent en grande partie des écoulements du noyau. En particulier, une variation d'une fraction de $\mathrm{ms}$ et de périodicité de l'ordre de six ans signe la présence d'ondes de torsion, comme illustré par la figure 6. Les écoulements du noyau sont ici reconstruits à partir de mesures magnétiques complètement indépendantes des données de durée du jour. Le bon accord entre ces observations et nos prédictions donne ainsi confiance dans les reconstitutions de la dynamique du noyau.

Le calcul du moment cinétique global de la Terre doit aussi prendre en compte les variations de son moment d'inertie aux

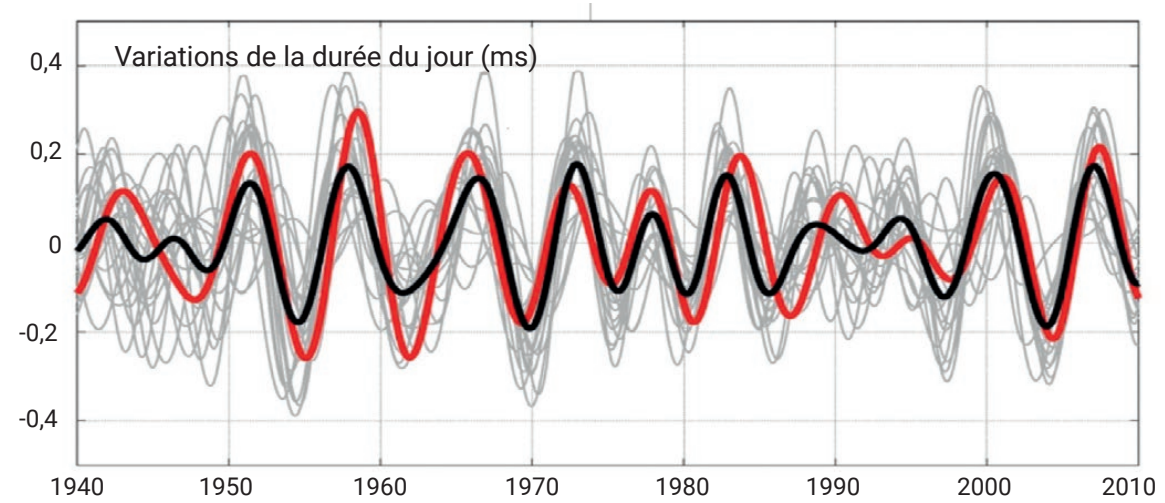

6. Variations de la durée du jour terrestre. Comparaison entre les composantes mesurées de période voisine de 6 ans (en rouge ; unités : ms) et les prédictions utilisant un ensemble d'écoulements du noyau terrestre, reconstitués à partir des mesures magnétiques (en gris, moyenne en noir) [6]. périodes interannuelles, dues à la redistribution à la surface terrestre des masses hydrologiques qui déforment notre planète. Or, les informations sur la dynamique du noyau issues des mesures magnétiques donnent en partie accès à ce bilan : le géomagnétisme pourrait donc aider à mieux connaitre les évolutions du bilan de masse du système océan-atmosphère-hydrosphère dues au changement climatique.

Les dynamiques des différentes enveloppes de notre planète ne peuvent pas être considérées indépendamment, comme en témoignent les variations de ses paramètres d'orientation tels que, par exemple, la durée du jour. Le signal magnétique est une des informations qui nous permet de passer de la magnétosphère au noyau.

\section{Remerciements}

Ce manuscrit a profité de discussions fructueuses avec, en particulier, J. Aubert, D. Cébron, D. Jault, G. Morard, H.-C. Nataf et N. Schaeffer. (a) $135 \mathrm{GPa}$ et $\approx 4000 \mathrm{~K}$ à la surface du noyau ; $330 \mathrm{GPa}$ et $5500 \mathrm{~K}$ à la surface de la graine solide, de rayon $1220 \mathrm{~km}$.

(b) Swarm est une mission de l'Agence spatiale européenne (ESA), qui a pour objectif d'étudier les variations spatiales et temporelles du champ géomagnétique, l'aimantation de la lithosphère, la conductivité électrique du manteau, ainsi que l'environnement ionosphérique de la Terre. Elle met en œuvre trois satellites identiques, qui ont été lancés en 2013.

(c) La diffusivité magnétique $\eta=1 /(\mu \sigma)$ est déterminée par la conductivité électrique $\sigma$ et la perméabilité magnétique $\mu$ du fluide.

(d) Le sodium est le métal le plus approprié, alliant une faible densité $\rho \approx 10^{3} \mathrm{~kg} / \mathrm{m}^{3}$ et une faible diffusivité magnétique $\eta \approx 0,1 \mathrm{~m}^{2} / \mathrm{s}$.

(e) Pour un processeur multicœurs, cette valeur représente la somme des temps de calcul des différents " cœurs" ("core" en anglais)

(f) La modélisation de la dynamique du noyau requiert une plus forte puissance de calcul que celles de l'océan ou de l'atmosphère, car pour ces dernières (i) l'épaisseur très fine autorise certaines approximations, (ii) des mesures in situ permettent de paramétrer certains phénomènes comme la taille des couches limites, et (iii) les modèles de turbulence - qui évitent de résoudre toutes les interactions aux petites échelles - sont plus aboutis.

\section{Références}

1 P. Cardin et P. Olson, "Experiments on core dynamics", dans Treatise on Geophysics, $2^{\mathrm{e}}$ édition, vol. 8, Core dynamics (2015) 317-339.

2• N. Schaeffer et al., "Turbulent geodynamo simulations: a leap towards Earth's core", Geophys. J. Int. 211 (2017) 1-29.

3• N. Gillet et al., "Fast torsional waves and strong magnetic field within the earth's core", Nature 465 (2010) 74-77.

4. J. Aubert et C.C. Finlay, "Geomagnetic jerks and rapid hydromagnetic waves focusing at earth's core surface", Nature Geoscience 12 (2019) 393-398.

5• F. Gerck et al., "Fast quasi-geostrophic magneto-coriolis modes in the earth's core", Geophys. Res. Lett. (2020) 2020GL090803.

6. N. Gillet et al., "Planetary gyre, time-dependent eddies, torsional waves, and equatorial jets at the Earth's core surface", J. Geophys. Res.: Solid Earth 120 (2015) 3991-4013. 